\title{
Cluster based Routing Protocols for IOT Application
}

\author{
Anna Merine George \\ Electronics \& Communication Engineering, Dayananda Sagar University Research Scholar, REVA University, \\ Bengaluru, India \\ E-mail: anna_thanku@yahoo.com
}

\author{
Dr. S.Y Kulkarni, Vice Chancellor \\ Electronics \& Communication Engineering, REVA University \\ E-mail: vc@ reva.edu.in
}

Received: 19 October 2018; Accepted: 20 March 2019; Published: 08 May 2019

\begin{abstract}
Extending the battery lifetime and reducing the power consumption using ultra low power sensor nodes and energy harvesting systems is essential to realize 50 billion IOT devices. Development of efficient routing algorithm is a critical aspect for reducing energy consumption and enhancing network lifetime. Clustering is a key technique used to enhance network lifetime. The lifetime of a wireless sensor network for Daylight Artificial Light Integrated Scheme is enhanced using Self Organizing Map (SOM) based clustering algorithm. A simulation of cluster based routing protocols like LEACH (Low Energy Adaptive Clustering Hierarchy), Fuzzy based LEACH and ANFIS based LEACH is also carried out using MATLAB software.
\end{abstract}

Index Terms-Battery lifetime, sensor nodes, SOM, LEACH, Building automation.

\section{INTRODUCTION}

A sensor network comprises of sensing module, processing component, transmission module and power module. Of these the radio communication is the most power hungry component and hence the radio component should adopt techniques that are energy efficient. The power supply mostly used is a rechargeable battery. Low power wireless technologies that meet demands of IOT include Thread, Wi-Fi HaLow, LoRa/LoRaWAN, Narrow Band Internet of Things (NB-IOT), Long Term Evolution (LTE) etc. Mesh networks are more suited for IOT applications due to their long range, flexibility and redundancy. System level and local clock speed management techniques like Dynamic Voltage and Frequency Scaling (DVFS) helps reduce power consumption [19].

Among the possible wireless solution, Bluetooth low energy has limited coverage range due to the lack of mesh networking support, and ZigBee is less developer friendly, since modifications on the ZigBee stack needs much effort. The Low Power Wireless Personal Area Networks (6LoWPAN) absorbs the features of mesh networking support and low-power consumption, and provides Internet Protocol (IP) support on resource and energy constrained devices [10]. The support for IP makes the 6LoWPAN protocol highly compatible with the existing Transmission Control Protocol/ Internet Protocol (TCP/IP) based Ethernet or Wifi networks. These attracting features make 6LoWPAN become an important candidate for implementation of future building automation.

Some of the ways to achieve better lifetime in sensor nodes include energy efficient routing, battery characteristics (recovery effects, thermal effects and discharge characteristics), operating the system in ultralow power standby mode, duty cycling the sensor and exercising power aware firmware [20].

Routing protocols are classified as Flat band routing, Hierarchical based routing (clustering) and location based routing (Geographic). Flat Band routing includes Flooding and Gossiping, Sensor Protocol for Information via Negotiation (SPIN), Directed Diffusion, Energy aware routing, Geocast Routing Protocol and Gradient Based Routing. Hierarchical protocols include LEACH (Low Energy Adaptive clustering Hierarchy), PEGASIS (Power Efficient Gathering in Sensor Information Systems), TEEN and APTEEN (Threshold sensitive Energy Efficient Sensor Network Protocol). Location Based Routing comprises of MECN (Min Energy Communication Network) \& SMECN, GEAR (Geographical Energy Aware Routing), GAF and HGAF (Geographical Adaptive Fidelity)

The lifetime of an IOT system would depend on the energy consumption of the sensor device. This includes the energy consumption due to the collective operations like sensing, processing, receiving and transmitting. By calculating the power consumption, one can evaluate the application's lifetime, become mindful of applications power consumption, help to implement techniques to improve the network's lifetime and to predict the time to replace the sensor node.

The aim of this work is to bring about wireless automation for lighting control in buildings using cluster 
based routing protocols. Lighting automation involves the use of environmental data to regulate the illuminance of a room such that maximum power savings are obtained while maintaining comfortable amount of light in the space at all times. Intelligent lighting control is still in its infancy with lot of scope for improvement in terms of the energy consumption of the individual nodes.

\section{RELATED WORKS}

In [1] Lee, Huang-Chen et al., have proposed several techniques for long range and outdoor wireless communication such as Sigfox, LoRa/LoRaWAN, NBIOT and LTE-M. Using LoRa mesh networking system an average $88.49 \%$ Packet Delivery Ratio (PDR) was obtained whereas star network topology achieved a PDR of $58.7 \%$ under similar settings. The advantage of the method includes collecting data from IOT sensors scattered over a campus sized area without using expensive $3 \mathrm{G} / 4 \mathrm{G}$ networks or laying cables for data transmission. The design using mesh network can lessen the problem of attenuation and increase PDR without deploying additional gateways. However the design focuses on wireless communication performance rather than on low power requirements. Cost in terms of power consumption is an issue for a battery powered node.

In [2] Lu, Xiaolin, et al have proposed methods to increase the battery life to 10 years using a platform called I3Mote. Network protocol running on I3Mote is 802.15.14 Direct Sequence Spread Spectrum (DSSS) PHY with Time Slotted Channel Hopping (TSCH). The techniques used to improve battery life include i) duty cycle optimization i.e If the sensor data are slowly changing based on previous data statistics, compress the data locally using algorithms like generalized linear precoding, ii) Protocol optimization iii) Wakeup Time Impact for radio - reduce the crystal oscillator start up time.

Hardware architecture for transmitter, channel and the receiver designed using SIMULINK is proposed in [3]. Bluetooth is used to carry out physical layer communication. Transmitter consists of Bluetooth Technology, Sensor signal stage, up sample to $64 \mathrm{~K}$ samples/s, payload Forward Error Correction (FEC) encode, Bluetooth Clock, Hop sequence generator, Encode and modulator. The medium consists of Additive White Gaussian Noise (AWGN) channel, path loss, $802.11 \mathrm{~b}$ interfaces and multi-port switch. Receiver consists of Hop sequence generator, demodulator \& decode, zero order hold, down sample to $8 \mathrm{~K}$ samples/s.

Bouarafa, Saleh et al., in [4] evaluated the impact of different network topologies and energy consumption of the network. To obtain effective routing schemes with reduced power consumption the topology utilizes the geographic and topological statistics of the network. Distribution of nodes in a network in a uniform manner leads to fading of energy of the nodes that are in proximity to the base station than the other nodes. Performance metrics used in this work is the number of active sensors in the network.
In this paper [5] Ouadou, Mourad, et al focused on Zigbee sensor networks based on IEEE 802.15.4 standard. The topology control is improved by proposing an efficient clustering algorithm based on Minimum Spanning Tree (MST). The proposed topology exhibited enhanced results than cluster tree and mesh topology in terms of energy consumption and networks lifetime. However the disadvantage for MST topology is the slow discharge process for router nodes.

The ultra-low power solution presented in [7] is designed with a custom antenna, a dedicated nano-power DC/DC converter, a low - power profile multi-sensor node and adopting a standard wireless communication protocol for IOT. The proposed design allows operation with negligible input power and permits operative distances up to $17 \mathrm{~m}$. Based on the current state of the harvesting module obtained through the interaction between the control signals of the power module and microcontroller, the microcontroller adapts its behavior to optimize the transmission rate. Temperature and humidity data are transmitted using the standard DASH7 protocol, which makes the node well suited with IOT infrastructure.

An innovative data driven approach to improve the use of power sources in WSNs is presented in [8]. A MultiLayer Perceptron is used to forecast the data, thereby achieving a power savings of $7.5 \%$. Continuous sampling by sensors is not employed. Temperature and humidity sensors are placed at three dissimilar positions namely a cold source, a warm source and a normal source.

Wireless energy harvesting (WEH) is one latest solution to enhance longevity and energy efficiency of IOT devices in lieu of lightweight communication protocols or low power radio transceivers. The WEH collects the radio waves that are transmitted using an antenna and transforms the RF energy into Direct Current (DC), and is then supplied to a sensor device. The sensor device uses an asynchronous duty cycling approach [9]. The Power management unit controls the storage of harvested energy and distributes the existing energy to different consumers. The energy harvesting increases the lifetime of the IOT device by $\sim 30$ percent and the Wakeup Radio (WUR) scheme boosts the lifetime by a further $\sim 110$ percent. Improving the performance of WEH systems at circuit and systems level needs more research. The current efficiency for sub-mW power level is $50 \%$. Solutions in the form of duty-cycling-aware middleware between Medium Access Control (MAC) and physical layer power management are highly anticipated.

Wireless communication is a crucial component of an IOT device from an energy perspective and improved efficiency can greatly improve device performance. Key challenges for power reduction in wireless systems at low duty cycles are in the joint optimization of protocols, RF circuits, leakage management and sleep-mode timing. Low voltage operation, a low-voltage Phase Lock Loop (PLL) developed with focus on the frequency divider, Voltage Controlled Oscillator (VCO) and charge pump circuits, extensive power gating and a negative gate biasing technique are used to reduce leakage currents. The CMOS based test-chip, manufactured in a $65 \mathrm{~nm}$ 
CMOS process has an efficiency of $43.7 \%$ while delivering a power of $+10.9 \mathrm{dBm}[11]$.

\section{SYSTEM DESCRIPTION}

The Architecture of Wireless Sensor Network (WSN) consist of data from the sensors that need to be logged on a periodic basis, actuators that are controlled based on a certain and Communication links as shown in Fig. 1. Communication across different locations will take place over the internet. There is a central gateway at each location connected to the internet over LAN.

Communication method between devices at a particular location is decided (ZigBEE/BLE/WiFi) considering power consumption and range [20].

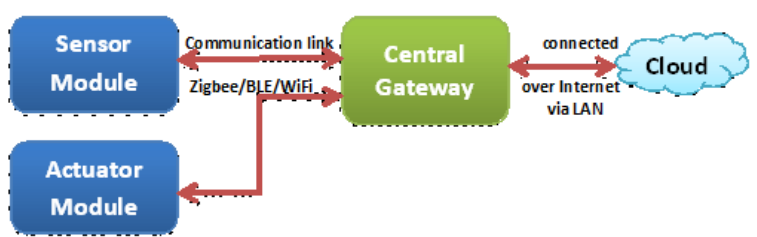

Fig.1 Architecture of WSN

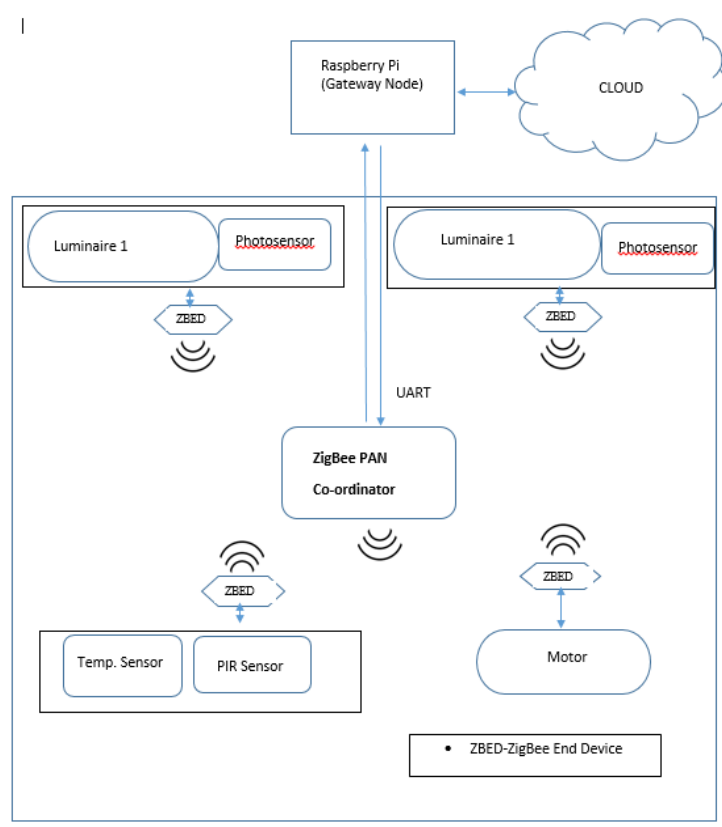

Fig.2. Proposed System

The proposed system shown in Fig. 2 consists of 100 sensor nodes installed in a floor of a building. The network is divided into four occupancy zones, each with its own Passive Infrared [PIR] Occupancy Sensors, two LED Luminaires and motorized window blinds [17,18]. One of the luminaires in each zone is placed close to a window, thus is exposed to more natural light than the other. Therefore the control signals required for each of these luminaires is different. To facilitate this, every luminaire is provided with a photo sensor of its own. The photo sensor circuit for each luminaire is calibrated separately depending on the maximum and minimum amount of luminous intensity that it is exposed to. Each luminaire and the associated photo sensor are connected to one XBee Module. One of the occupancy sensor nodes will also have a temperature sensor mounted on it. The temperature sensor and the PIR occupancy sensor are both interfaced with another XBee Module. A sixth XBee Module acts as the ZigBee PAN coordinator for this Wireless Sensor Network. All the other XBees periodically update the PAN coordinator with the data from the sensors attached to them.

The different routing protocols considered include:

\section{A. LEACH (Low Energy Adaptive Clustering Hierarchy)}

It is a clustering algorithm for reducing the power consumption of WSN. In this algorithm, a random rotation of high-energy Cluster Heads $(\mathrm{CH})$ position is used to give a chance for all sensors to act as $\mathrm{CHs}$ and to prevent the battery depletion and dying of individual sensors. Fig. 3 shows the radio dissipation model. The LEACH algorithm is divided into two phases namely (i) a setup phase to arrange the network into clusters, and (ii) a steady-state phase for data aggregation, compression, and transmission to the sink [13]. The algorithm minimizes energy consumption by (a) reducing the communication cost between sensors and their cluster heads (b) turning off non-head nodes whenever possible. LEACH exploits single-hop routing where each node transmits directly to the cluster-head and the sink.

Clusters are organized and CHS are selected based on threshold value $T(n)$

$$
T(n)=\left\{\begin{array}{cr}
\frac{p}{1-p\left(r \bmod \frac{1}{p}\right)} & \text { if } n \in G \\
0 & \text { Otherwise }
\end{array}\right.
$$

Where $\mathrm{p}=$ desired percentage of $\mathrm{CHs}, \mathrm{r}=$ current round and $\mathrm{G}=$ set of nodes that have not been cluster heads in the last $\frac{1}{p}$ rounds.

Each node selects a random number between 0 and 1 and compares to $T(n)$. If the number is lower than a threshold, node becomes cluster head.

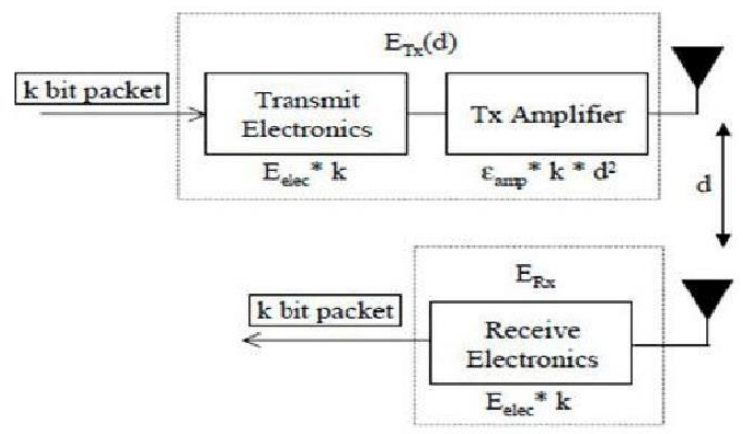

Fig.3. Radio Energy Dissipation Model [15]

LEACH algorithm reduces energy dissipation by a factor of 7 as compared to direct communication and reduces energy dissipation by a factor of $4-8$ when compared to the minimum transmission energy routing protocol [14]. 


\section{B. Improved LEACH protocol using Fuzzy Logic Approach}

In this protocol a priority value is assigned for each cluster head $(\mathrm{CH})$ based on initial energy, distance from base station (BS) and data transmission rate [12]. The BS moves towards each $\mathrm{CH}$ by a distance proportional to that CH's priority. This is accomplished by setting up a vector between the BS and each $\mathrm{CH}$, with the vector's magnitude equal to that $\mathrm{CH}$ 's priority multiplied by a constant step size.

\section{ANFIS based LEACH algorithm}

Adaptive Neuro Fuzzy Inference System (ANFIS) exploits a hybrid training method to generate fuzzy rules in accordance to a given input - output datasets. ANFIS uses neural learning rules to tune the parameters and structure of a Fuzzy Inference System. Training procedure for ANFIS is obtained by batch learning technique [16].

The input-output training dataset for ANFIS model was developed from the Fuzzy based LEACH model.

\section{Self-Organizing Maps (SOM)}

The Self-Organizing Map (SOM) is an unsupervised learning algorithm used for cluster head selection in order to maximize lifetime of WSN. Here the neuron is represented by an $n$ - dimensional weight vector. Weight vectors make connections with the input layer to output layer called map or competitive layer. Every input vector stimulates a neuron in output layer (called winner neuron) based on its similarity. The similarity is usually computed by the Euclidian distance of two vectors.[15]

\section{SimULATION RESULTS}

QualNet is simulation software used to study and compare the efficiency of various routing protocols. An energy model analysis of all the nodes in the Idle, Transmit and Receive modes is carried out using the same. It is observed that the mesh topology offers the minimum power consumption in idle mode followed by the tree and star topologies. This result is constant over both AdHoc on Demand Distance Vector Routing (AODV) and Dynamic Source Routing (DSR) protocols.

The average amount of power transmitted is also found to be minimal in the mesh topology and the tree topology almost resembles the mesh topology in terms of power used in the transmit mode when the AODV protocol is used. With the DSR protocol, the tree topology spends more power. The power used in the star topology is still significantly higher.

In the receive mode, it is found that the star and mesh topologies spend very less power for both AODV and DSR protocols. The tree protocol though consumes a significantly greater amount of power in both cases. Table 1 shows a comparison of various wireless technologies.
Table 1. Shows a Comparison of Various Wireless Technologies, their Topology and Battery Life.

\begin{tabular}{|l|l|l|l|}
\hline Standard & Topology & $\begin{array}{l}\text { Battery } \\
\text { Life (days) }\end{array}$ & $\begin{array}{l}\text { Range } \\
(\mathrm{m})\end{array}$ \\
\hline Zigbee & Mesh & $100-1000+$ & $10-100$ \\
\hline 6LoWPAN & Mesh & $100-365+$ & $1-100$ \\
\hline WirelessHART & Mesh & $760+$ & $1-100$ \\
\hline ISA 100.11a & Mesh, star & $1000+$ & 100 \\
\hline
\end{tabular}

The number of dead nodes (shown in red), number of alive nodes (blue line) and average energy of each node after 100 iterations using LEACH algorithm are shown in Fig. 4 and Fig. 5 respectively.

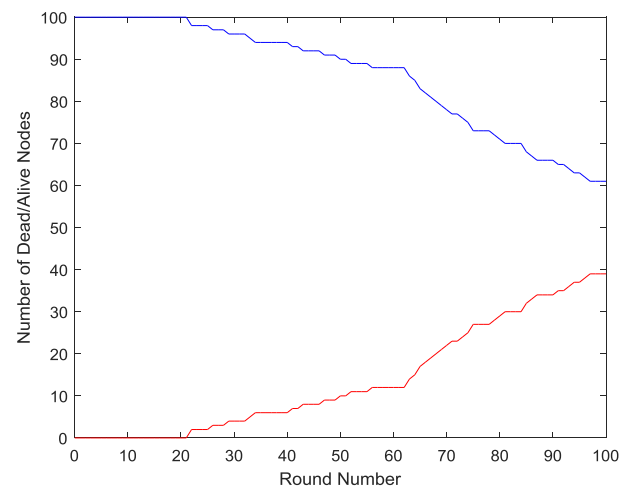

Fig.4. Number of Dead/Alive nodes using LEACH Algorithm

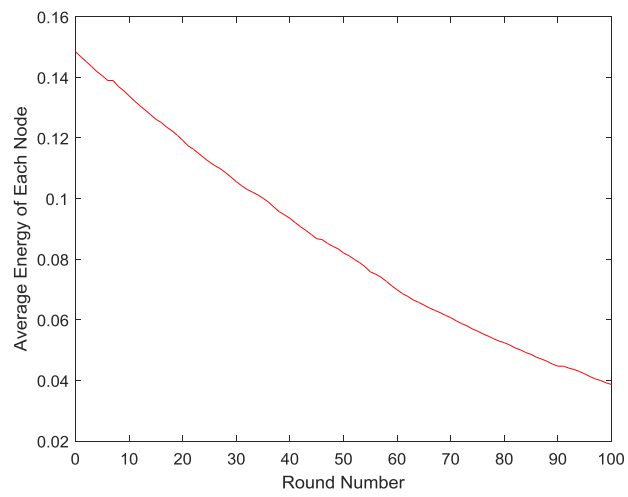

Fig.5. Average Energy of each node using LEACH
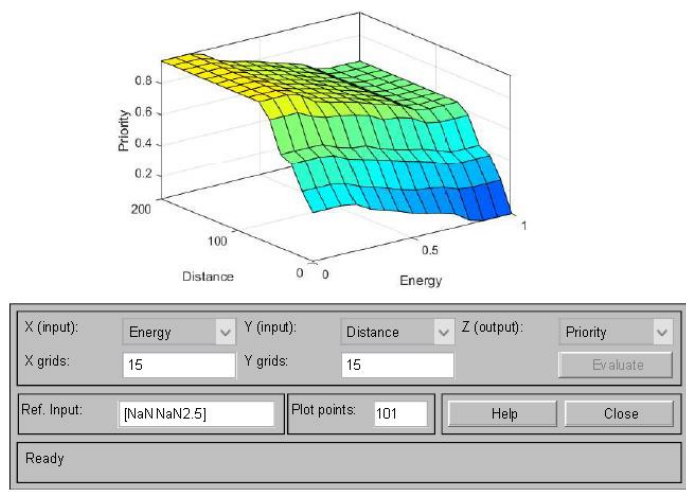

Fig.6. Surface Plot for Cluster Head Priority using Fuzzy based LEACH 
The surface plot obtained for cluster head priority with inputs as Initial energy of nodes, distance to base station and rate of transmission (fixed to 2.5 in plot) using Fuzzy based LEACH is shown in Fig.6. Using Mamadani based Fuzzy Inference System (FIS) 27 rules are written using fuzzy toolbox of MATLAB.

Table 2. Priority Value for Different Cluster Heads using Fuzzy based LEACH

\begin{tabular}{|c|c|c|c|}
\hline $\begin{array}{c}\text { Initial } \\
\text { Energy }(\mathbf{J})\end{array}$ & $\begin{array}{c}\text { Distance to } \\
\text { Base Station } \\
(\mathrm{m})\end{array}$ & $\begin{array}{c}\text { Data transfer } \\
\text { Rate }(\text { Kbps })\end{array}$ & $\begin{array}{c}\text { Priority } \\
\text { using FIS }\end{array}$ \\
\hline 0.2 & 45 & 4 & 0.691 \\
\hline 0.2 & 15 & 4 & 0.558 \\
\hline 0.2 & 128 & 3 & 0.946 \\
\hline 0.5 & 10 & 2 & 0.213 \\
\hline 0.8 & 80 & 4 & 0.633 \\
\hline 0.8 & 10 & 1 & 0.217 \\
\hline
\end{tabular}

The surface plot obtained for cluster head priority with inputs as Initial energy of nodes, distance to base station and rate of transmission (fixed to 2.5 in plot) using ANFIS based LEACH is shown in Fig.7.

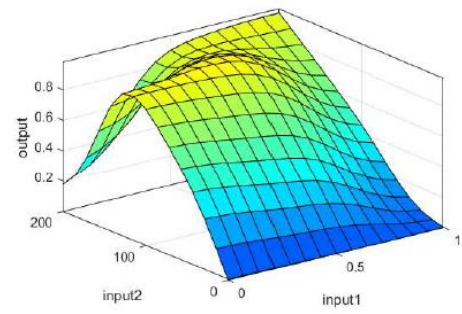

\begin{tabular}{|c|c|c|c|c|c|}
\hline \multirow{2}{*}{$\begin{array}{l}x \text { (input): } \\
x \text { grids: }\end{array}$} & input1 & \multirow{2}{*}{$\begin{array}{l}\checkmark Y \text { (input): } \\
Y \text { grids: }\end{array}$} & input2 & \multirow{2}{*}{$\checkmark Z$ (output): } & \multirow{2}{*}{\begin{tabular}{|c|} 
output \\
Evaluate \\
\end{tabular}} \\
\hline & 15 & & 15 & & \\
\hline Ref. Input: & [NaN NaN2.5] & & nts: 101 & Help & Close \\
\hline
\end{tabular}

Fig.7. Surface Plot for Cluster head Priority using ANFIS based LEACH

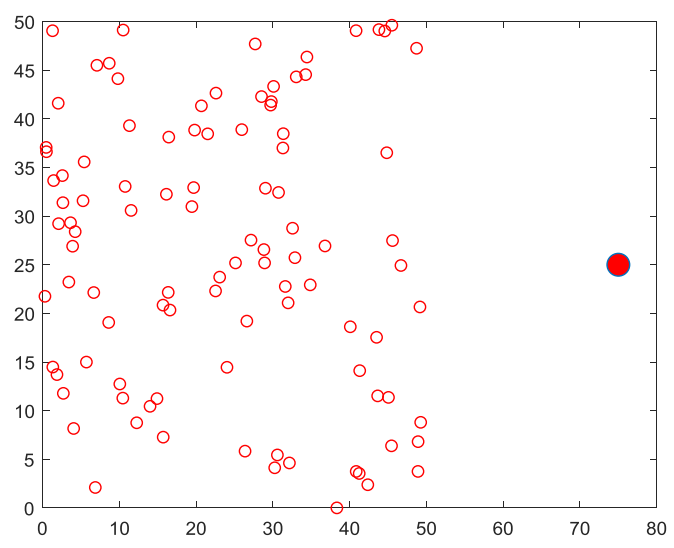

Fig.8. 100 Sensors Placed in $\mathrm{x}-\mathrm{y}$ Plane along with sink Node

In SOM based clustering three cases considered are as follows:

\section{A. SOM Based Clustering (Distance as Metric)}

The clusters are created so as to minimize the sum of distance between the sensor and the sensor which is cluster head. 100 sensors are placed evenly on $x-y$ plane and each sensor is given a fixed amount of initial power (1000 in this case) as shown in Fig 8. In fig. 8 the shaded red dot represents the sink node.

The number of clusters has been fixed to 4 . The remaining power in each node while the sensors are placed on the $\mathrm{x}, \mathrm{y}$ plane is as shown in Fig. 9

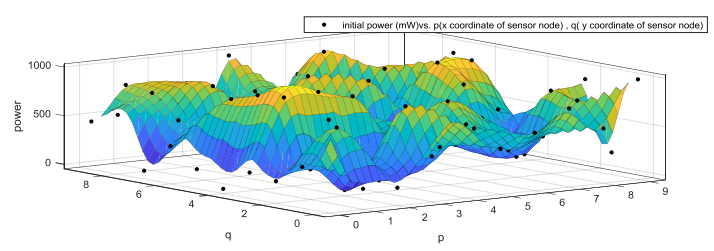

Fig.9. Remaining Power in each Sensor Node

The results obtained from Nntraintool GUI for case A is as shown in Fig.10 and Fig.11.

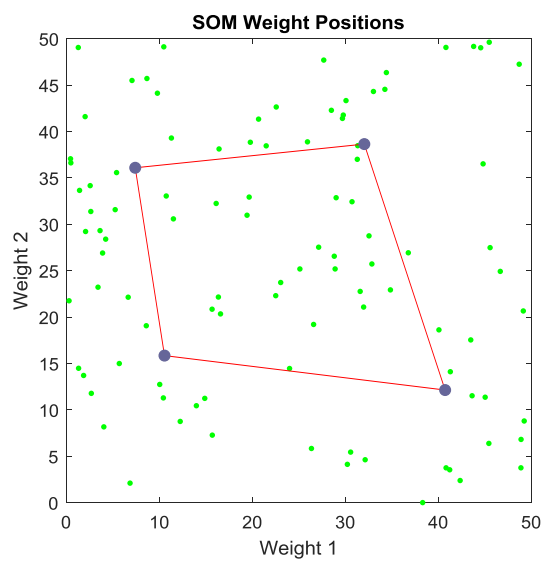

Fig.10. SOM Weight Positions

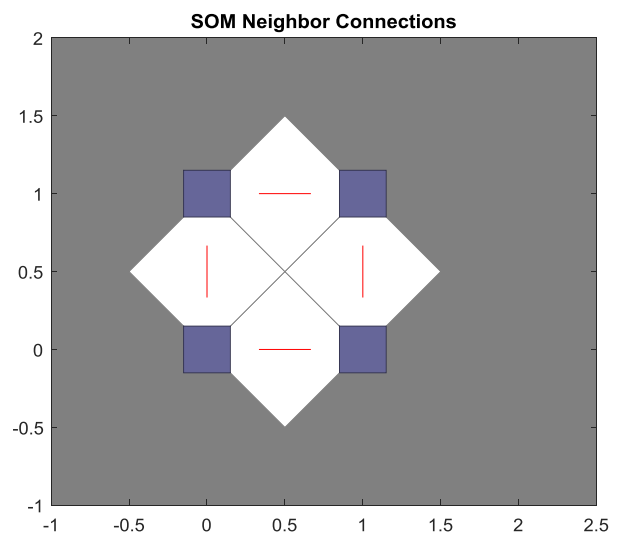

Fig.11. SOM Neighbor Connections

SOM Neighbor distances - It shows the distance of sensors from cluster centers as computed using distance function and the neighborhood of each cluster centers are shaded in different colors.

SOM Weight Positions- The cluster centers are shown at their weight vector (using them as position vector) along with all the sensors in the WSN. 


\section{B. SOM Clustering (Power and Distance as Metric)}

MATLAB is used to find the cluster id of each sensor and the sensor for which the objective function (composed of power and distance from cluster center) is minimum is chosen as cluster head. Hence as the time passes, it can be observed that the power is consumed by all the sensors at approximately the same rate.

The results obtained from Nntraintool GUI for case B is as shown in Fig.12 and Fig.13.

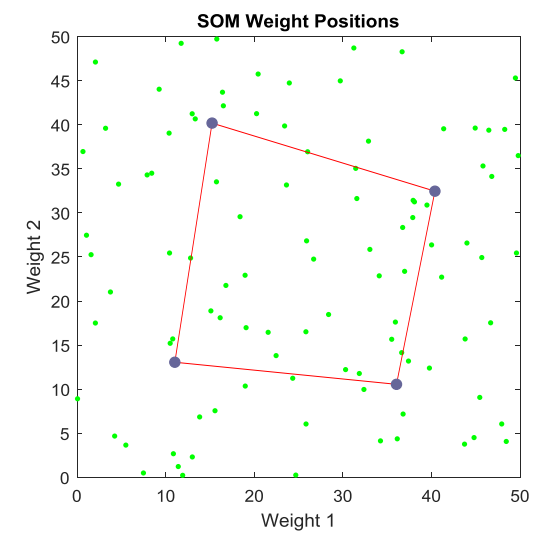

Fig.12. SOM Weight Positions

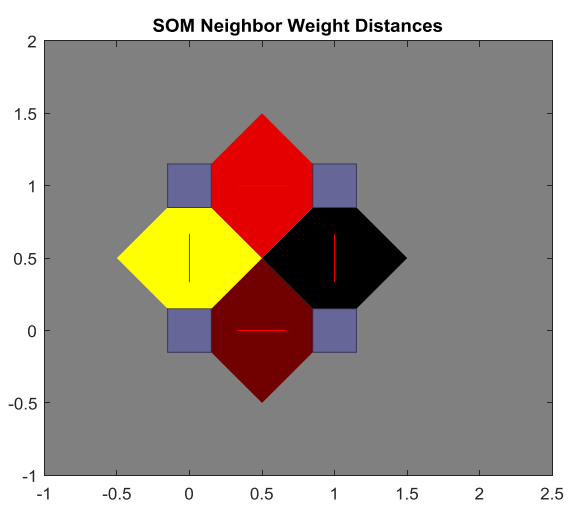

Fig.13. SOM Neighbor Connections

\section{Recalculating Clusters Iteratively after Getting Cluster using SOM Initially.}

Initially, cluster is evaluated using SOM which uses distance as metric. The cluster to which each sensor belongs to is known. Now, cluster head is chosen such that the sensor for which the objective functions which constitutes remaining power and the distance from geometrical centroid of cluster to the sensor, is minimized.

After this cluster is recalculated and each sensor is assigned to the cluster whose cluster head is closest to it. Cluster heads and then the cluster is computed iteratively.

The results obtained from Nntraintool GUI for case C is as shown in Fig. 14.

The cluster head index, size of each cluster and power of cluster for three cases of SOM algorithm is summarized in table 3 .

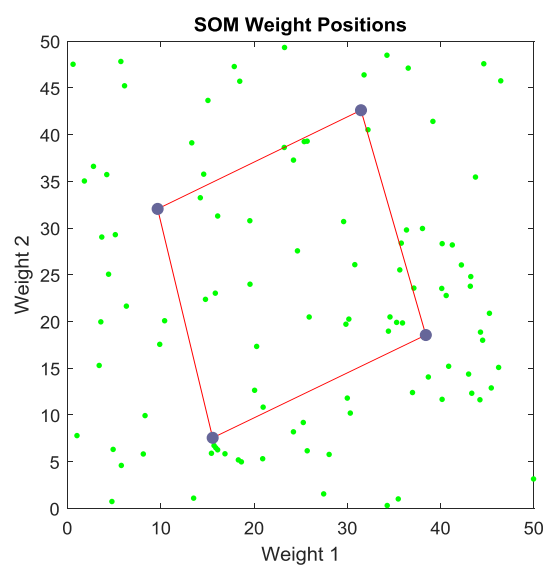

Fig.14. SOM Weight Positions

Table 3. Summary of Cluster Head Index and Power of Clusters for three cases of SOM Algorithm

\begin{tabular}{|c|c|c|c|}
\hline & Case A & Case B & Case C \\
\hline $\begin{array}{l}\text { Cluster head } \\
\text { ID's }\end{array}$ & $23,28,73,78$ & $33,10,95,87$ & $33,10,95,87$ \\
\hline $\begin{array}{l}\text { Size of each } \\
\text { Cluster }\end{array}$ & $25,25,25,25$ & $25,25,25,25$ & $40,18,15,27$ \\
\hline $\begin{array}{l}\text { Power of } \\
\text { cluster } \\
\text { heads }(\mathrm{mW})\end{array}$ & $\begin{array}{l}23.0421 \\
536.3906 \\
534.2111 \\
114.8000\end{array}$ & $\begin{array}{l}23.0421 \\
536.3906 \\
534.2111 \\
114.8000\end{array}$ & $\begin{array}{l}23.0421 \\
536.3906 \\
534.2111 \\
114.8000\end{array}$ \\
\hline $\begin{array}{l}\text { Sum of } \\
\text { power of } \\
\text { each clusters } \\
(\mathrm{mW})\end{array}$ & $\begin{array}{l}1.14 \mathrm{e}+04 \\
1.2403 \mathrm{e}+04 \\
1.3392 \mathrm{e}+04 \\
1.3005 \mathrm{e}+04\end{array}$ & $\begin{array}{l}1.14 \mathrm{e}+04 \\
1.2403 \mathrm{e}+04 \\
1.3392 \mathrm{e}+04 \\
1.3005 \mathrm{e}+04\end{array}$ & $\begin{array}{l}1.7519 \mathrm{e}+04 \\
9.0062 \mathrm{e}+03 \\
8.8148 \mathrm{e}+03 \\
1.4860 \mathrm{e}+04\end{array}$ \\
\hline
\end{tabular}

\section{CONCLUSIONS}

The IOT nodes are mostly deployed in remote environments where replacement of battery becomes impossible every time. Clustering based routing protocols is important to enhance the lifetime of IOT networks. Appropriate cluster head selection is a challenging issue that can reduce energy consumption. Different cluster head selection methods considered in this paper include LEACH, Fuzzy Based LEACH, ANFIS based LEACH and SOM based clustering.

\section{REFERENCES}

[1] Lee, Huang-Chen, and Kai-Hsiang Ke. "Monitoring of Large-Area IOT Sensors Using a LoRa Wireless Mesh Network System: Design and Evaluation." IEEE Transactions on Instrumentation and Measurement (2018).

[2] Lu, Xiaolin, et al. "Reaching 10-years of battery life for industrial IoT wireless sensor networks." VLSI Circuits, 2017 Symposium on. IEEE, 2017.

[3] Ali, Qutaiba Ibrahem, Akram Abdulmaowjod, and Hussein Mahmood Mohammed. "Simulation \& performance study of wireless sensor network (WSN) using MATLAB." Energy, Power and Control (EPC-IQ), 2010 1st International Conference on. IEEE, 2010.

[4] Bouarafa, Saleh, Rachid Saadane, and Driss Aboutajdine. "Reduction of energy consumption in WSN using the Generalized Pythagorean Theorem." Multimedia Computing and Systems (ICMCS), 2016 5th International Conference on. IEEE, 2016. 
[5] Ouadou, Mourad, et al. "Improved Cluster-tree Topology Adapted for Indoor environement in Zigbee Sensor Network." Procedia Computer Science 94 (2016): 272279.

[6] Silva, Agnelo, Mingyan Liu, and Mahta Moghaddam. "Power-management techniques for wireless sensor networks and similar low-power communication devices based on nonrechargeable batteries." Journal of Computer Networks and Communications 2012 (2012).

[7] Pizzotti, Matteo, et al. "A Long-Distance RF-Powered Sensor Node with Adaptive Power Management for IoT Applications." Sensors 17.8 (2017): 1732.

[8] Aram, Siamak, Luca Mesin, and Eros Pasero. "Improving lifetime in wireless sensor networks using neural data prediction." Computer Applications \& Research (WSCAR), 2014 World Symposium on. IEEE, 2014.

[9] Kamalinejad, Pouya, et al. "Wireless energy harvesting for the Internet of Things." IEEE Communications Magazine 53.6 (2015): 102-108.

[10] Min, Xiaoyu. "Experimental study on low power wireless sensor network protocols with native IP connectivity for building automation." (2016).

[11] Paidimarri, Arun. Circuits and protocols for low duty cycle wireless systems. Diss. Massachusetts Institute of Technology, 2016.

[12] Nayak, Padmalaya, and Anurag Devulapalli. "A fuzzy logic-based clustering algorithm for WSN to extend the network lifetime." IEEE sensors journal 16.1 (2016): 137144.

[13] Tayeb, Shahab, Miresmaeil Mirnabibaboli, and Shahram Latifi. "Cluster Head Energy Optimization in Wireless Sensor Networks." Software Networking 2018.1 (2018): 137-162.

[14] Akkaya, Kemal and Mohamed F. Younis. "A survey on routing protocols for wireless sensor networks." Ad Hoc Networks 3 (2005): 325-349.

[15] Cordina, Mario, and Carl James Debono. "Maximizing the lifetime of wireless sensor networks through intelligent clustering and data reduction techniques." Wireless Communications and Networking Conference, 2009. WCNC 2009. IEEE. IEEE, 2009.

[16] George, Anna Merine, et al. "Hardware in Loop Simulation of an Adaptive Traffic Light Control System." International Journal of Applied Engineering Research 10.19 (2015): 39999-40004.
[17] Kurian, Ciji Pearl, et al. "Fuzzy Logic based window blind controller maximizing visual comfort, thermal comfort and energy conservation suitable for tropical climate." Journal of the Institution of Engineers (India): Architectural Engineering Division 89. APRIL (2008): 14-22.

[18] Varghese, S. G., et al. "Climate model based test workbench for daylight-artificial light integration." Lighting Research \& Technology (2018): 1477153518792586.

[19] Anna Merine George, Dr. S.Y Kulkarni, "Performance of Power Converters for Ultra Low Power Systems: A Review", 2018 Second International Conference on Advances in Electronics, Computer and Communications (ICAECC-2018), held in REVA University between February 9-10 2018, 978-1-5386-3785-2, 2018 IEEE.

[20] Anna Merine George, Kulkarni S.Y, George V.I, “A Survey on Ultra Low Power Design Techniques for IOT Application", Current Trends in Information Technology, 2017; 7(3) pp 9-16.

\section{Authors' Profiles}

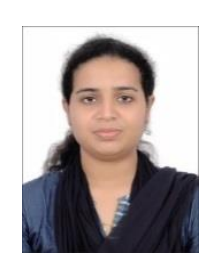

Mrs. Anna Merine George is a Research Scholar in the Department of Electronics \& Communication Engg at Reva University, Bengaluru. She completed her M. Tech in Digital Electronics \& Advanced Communication from M.I.T Manipal in 2015. She has around 4.5 years of teaching experience. Her teaching and research interest includes signal processing, VLSI and embedded system. She has 5 journal publications and 7 conference papers to her record.

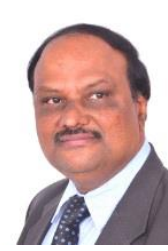

Dr. S.Y. Kulkarni is the Vice Chancellor of Reva University, Bengaluru. Prior to this he was principal at M.S.Ramaiah Institute of Technology, Bangalore and NMAM Institute of Technology, Nitte. His area of research interest includes VLSI.

How to cite this paper: Anna Merine George, S.Y Kulkarni, Vice Chancellor,"Cluster based Routing Protocols for IOT Application", International Journal of Computer Network and Information Security(IJCNIS), Vol.11, No.5, pp.4349, 2019.DOI: 10.5815/ijcnis.2019.05.06 